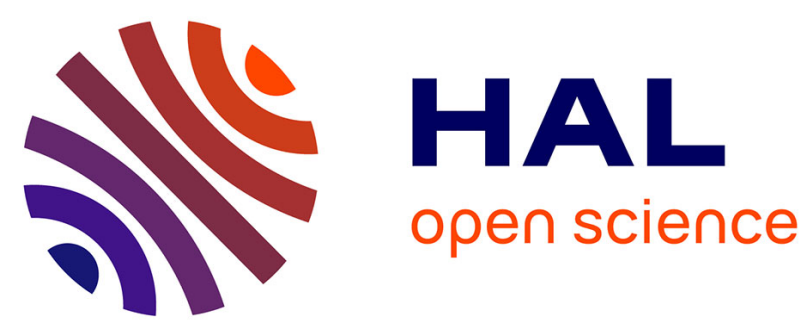

\title{
Toward a Robot Computing an Online Estimation of the Quality of its Interaction with its Human Partner
}

\author{
Amandine Mayima, Aurélie Clodic, Rachid Alami
}

\section{To cite this version:}

Amandine Mayima, Aurélie Clodic, Rachid Alami. Toward a Robot Computing an Online Estimation of the Quality of its Interaction with its Human Partner. 2020 29th IEEE International Conference on Robot and Human Interactive Communication (RO-MAN), Aug 2020, (Virtual Conference ), Italy. 10.1109/RO-MAN47096.2020.9223464 . hal-02910899

\section{HAL Id: hal-02910899 \\ https://hal.laas.fr/hal-02910899}

Submitted on 3 Aug 2020

HAL is a multi-disciplinary open access archive for the deposit and dissemination of scientific research documents, whether they are published or not. The documents may come from teaching and research institutions in France or abroad, or from public or private research centers.
L'archive ouverte pluridisciplinaire HAL, est destinée au dépôt et à la diffusion de documents scientifiques de niveau recherche, publiés ou non, émanant des établissements d'enseignement et de recherche français ou étrangers, des laboratoires publics ou privés. 


\title{
Toward a Robot Computing an Online Estimation of the Quality of its Interaction with its Human Partner
}

\author{
Amandine Mayima $^{1}$, Aurélie Clodic ${ }^{1,2}$ and Rachid Alami ${ }^{1,2}$
}

\begin{abstract}
When we perform a collaborative task with another human, we are able to tell, to a certain extent, how things are going and more precisely if things are going well or not. This knowledge allows us to adapt our behavior. Therefore, we think it is desirable to provide robots with means to measure in real-time the Quality of the Interaction with their human partners. To make this possible, we propose a model and a set of metrics targeting the evaluation of the QoI in collaborative tasks through the measure of the human engagement and the online task effectiveness. These model and metrics have been implemented and tested within the high-level controller of an entertainment robot deployed in a mall. The first results show significant differences in the computed QoI when in interaction with a fully compliant human, a confused human and a noncooperative one.
\end{abstract}

\section{INTRODUCTION}

To devise robot control systems endowing the robot with the ability to execute its share of the work while adapting to contingencies, particularly those caused by human behaviours, is one of the challenges that cognitive and interactive robot designers are facing. To handle it, human-aware models and algorithms have been proposed (e.g. a model to handle perspective-taking issues [1]) as well as architectures (e.g. [2]). However, surprisingly and interestingly, very little has been done to allow the robot to constantly estimate if the collaborative task is proceeding well in terms of Quality of Interaction.

When we perform a collaborative task with another human, we are able to tell, to a certain extent, how things are going and more precisely if things are going well or not. In other words, we are able to evaluate our own contribution but also the one of the collaborator we are working with. This knowledge allows us to adapt our behavior. Therefore, we think it is desirable to equip robots with such an ability, i.e. to provide them means to measure the Quality of the Interaction. In this way, they would be able to state if the Quality of the Interaction is satisfying or not, to analyse the changes over time and to adapt their behavior accordingly.

Although the concept of Quality of Interaction (QoI) is quite abstract, [3] shows that when it is measured by human observers, the inter-observer reliability of the concept is quite high. Multiple methods to evaluate robots performance and human-robot interactions exist but in most cases, the evaluation is achieved by humans and after the performance. Only a few frameworks try to make the robot evaluate the

\footnotetext{
${ }^{1}$ Authors are with LAAS-CNRS, Université de Toulouse, CNRS, Toulouse, France firstname. surnamed laas.fr

${ }^{2}$ Authors are with Artificial and Natural Intelligence Toulouse Institute (ANITI)
}

quality of the ongoing interaction. For example, in [4], one of the proposed metrics measures "the robot contribution to the fluency of the interaction" whereas in our case we are (also) interested in "the human contribution to the fluency of the interaction". This is precisely the topic of this paper.

We define the Quality of Interaction in Section III. Then, we propose a set of metrics in Section IV, targeting the evaluation of the QoI in collaborative tasks through the measure of the human engagement and the online task effectiveness. The proposed metrics may be used to evaluate the QoI at the task or action levels. Therefore, if an action is not going well it has an impact on the task QoI. We define metrics allowing us to have an estimation of the human engagement which are human contribution to the goal (i.e., how well the human executes the actions they are expected to do) and fulfilling robot expectations (i.e., how the human reacts to the robot actions). For the online task effectiveness measure, we propose metrics which we coin variation of the distance to goal for geometric-based subtasks/actions (i.e., to measure, during a motion, how the agent is getting closer to the target position over time), time to goal variation (i.e., to measure the progress of a task or an action towards its goal based on the estimation of the time needed to reach it) and On-Time-Execution (i.e., to represent the degradation of the quality of the task execution when its duration exceeds a certain time).

We present in Section VI an implementation of the model and metrics, the QoI Evaluator, within the high-level controller of a robot deployed in a mall. Finally, in Section VII, we provide and discuss a comparison of the QoI computed by the robot when it is dealing with a fully compliant human, a confused human and a not-compliant one.

\section{RELATED WORK}

Inspired from the evaluation methods from HCI, the field of Human-Robot Interaction (HRI) has elaborated its own methods to evaluate robotic systems and human interactions with the robot. The evaluation of such interactions raises three main questions: what to evaluate (e.g. task effectiveness), how to evaluate in order to choose a measure (e.g. using time or the number of errors as a measure) and why to evaluate (e.g. being able to compare with other systems). The "what to evaluate" of a system will vary according to the "why to". Metrics for HRI can be divided in two categories: subjective and objective metrics. For both categories, most of the contributions in the literature deal with evaluations and analysis after the interaction and not during the interaction. 
Subjective measures are most often questionnaires to users after their interaction with the robot such as in [5] where users were asked to evaluate individual system components (personal attention system, speech processing, dialog management, robot internal status) and then to give their preferences concerning the robot. They can also be complemented by observations such as in [6]. In [7] the authors proposed a theoretical framework inspired from HCI and user experiences. [8] presented a set of benchmarks divided into three categories: benchmarks dealing with evaluation of robot performance based on safety and scalability, benchmarks based on the evaluation of robot autonomy, imitation and privacy and finally benchmarks concerning robot social abilities and understanding of domain.

[4] used a combination of subjective and objective measures as well to evaluate the fluency in human-robot collaboration. They admitted that the notion of fluency is not well defined and somewhat vague but claimed it can be assessed and recognized when compared to non-fluent scenario. They built a questionnaire to assess how people perceive the collaboration with the robot. They also proposed a list of objective metrics, only based on duration measures, designed to be quite general: robot idle time, human idle time, concurrent activity, functional delay. [9] proposed a set of metrics to specifically evaluate navigation functions, perception abilities, robot management by a human operator, manipulation tasks and social functionalities. They also proposed a set of task-independent metrics, as in [10], used to assess the system performance such as the quantitative performance of a task execution based on the effectiveness and the efficiency. Finally, they proposed metrics to evaluate the robot performance based on its self-awareness, its awareness about the human and its autonomy. Their work is very thorough and inspiring but does not target the evaluation of the quality of an on-going interaction.

Some papers assess human-robot interactions with objective metrics based on the measure of human states. In [11], they proposed a framework allowing the robot to perceive and evaluate in real-time the affective state and the engagement state of the people it is interacting with. In [12], a bio-instrumentation system is proposed in order to evaluate human stress in real-time by measuring physiological parameters such as respiration, heart rate, perspiration, pulse wave and arm motion. In [13], these parameters are used to compare various ways to implement a handover joint action between a human and a robot.

Only a few papers propose a scheme which "closes the loop" between evaluation and the adaptation of the robot behaviour. In [11], the evaluation of the affective and engagement states of the human is used by the robot decision making processes. In [12], the robot executes a motion specifically designed to decrease human stress when assessed too high.

In summary, while a substantial number of studies have been devoted to the evaluation of interactive robots by humans, there is a lack of robotic frameworks allowing the robot to evaluate in real-time the on-going interaction, based on multiple metrics. We claim that such an ability is very important and should strongly influence the situation assessment as well as the decisional abilities of interactive and collaborative robots.

\section{The QuAlity OF InTERACTION}

As mentioned above, only a small number of contributions consider the quality of the interaction from the robot point of view and there is no established model in the literature. Having this ability would enhance its decision making process. For example, if the robot detects that the QoI starts to drop, it can choose to change some modalities such as the language in which it communicates with the human, the volume of its speakers, or the parameters of its planners. Also, a very poor performance all along a task could allow to assess that the human is not really engaged in the interaction, or is trying to play the robot. In such situation, the robot might better disengage. Finally, off-line, the developer may use the task logs to improve their design. As mentioned above, we focus in this paper only on the quality evaluation process. We have tried to define a set of metrics serving our purpose but, this is by no means exhaustive, and other metrics and parameters could be added later. This should be seen as a toolbox among which it is possible to pick the wanted metrics according to the tasks or contexts.

Definition: We define the Quality of Interaction (QoI) as a value computed in real-time based on a set of metrics, as in [14]. It can be impacted by other metrics seen as bonus or penalties. Moreover, we consider the QoI at the action level as an element to be taken into account at the task level.

All along a task, task QoI and action QoI are computed at a given rate. The QoI value of both levels is a score between 1 - for a good quality - and -1 - for a bad one. Metrics are divided in three categories:

- mep $\in\{0,1\}$ if it can only have a positive effect on the evaluation

- men $\in\{-1,0\}$ if a metric can only have a negative effect on the evaluation

- $m e \in\{-1,1\}$ if a metric can have a positive or a negative effect

Defined by the designer accordingly to the needs and context, a metric can belong to one category for a given application and to another category for another application.

The evaluation of the quality $Q o I_{l}$ is defined as:

$$
\left\{\begin{array}{l}
Q o I_{l}=\frac{\sum_{i=1}^{x} w_{i} m e_{i}}{\sum_{i=1}^{x} w_{i}}+w_{o} \frac{\sum_{i=1}^{y} w n_{i} m e n_{i}+\sum_{i=y}^{z} w p_{i} m e p_{i}}{\sum_{i=1}^{y} w n_{i}+\sum_{i=y}^{z} w p_{i}} \\
Q o I_{l, \max }=1 \\
Q o I_{l, \min }=-1
\end{array}\right.
$$

with $w_{i}, w p_{i}, w n_{i}$ respectively the corresponding designerset weights of $m e_{i}$, mep $_{i}$, men $_{i}$ and $l \in\left\{\right.$ task $_{k}$, action $\left._{m}\right\}$ the level for which the QoI is evaluated.

The left part of the + sign is a weighted mean of the metrics included between -1 and 1. The right part is a weighted mean of the metrics seen as bonus or penalty, 
weighted with $w_{0}$ to be able to adjust its influence on the left part. In such a way, if there is no bonus or penalty, they have no impact on the other metrics, and the other way round they can increase or decrease the QoI.

\section{A SET OF METRICS}

Two of our main concerns were how to measure the human engagement and the effectiveness in human-robot joint actions. Both concepts are not easily measurable but we claim to be able to give estimations. As we said previously, works such as [9][10] propose sets of metrics widely used but they do not target the evaluation of the quality of an on-going interaction. Therefore, our goal was to conceive metrics targeting our needs. It led to the set of metrics presented below, a tool to measure the QoI. This set is not exhaustive and will be extended in future work but it gave promising results as we show with our implementation in Section VI.

\section{A. Metrics related to human engagement}

We define here below two metrics which can be pertinent with respect to the engagement of the human partner.

1) Human contribution to the goal: When performing a task, there are actions to perform or situations to satisfy which need the human contribution in order to advance towards the shared goal. A good and very promising indicator could be the robot ability to evaluate how well the human does what they are expected to do. To the best of our knowledge, there is no general method to estimate it. For the moment, we have implemented a simple estimator based on the fulfilment of robot expectations and the "distance" between what is expected and what happens.

2) Fulfilling robot expectations: During the execution of some of the robot actions, the human is expected to behave in a certain way. Then, the robot monitors the human behavior to check if they are acting as needed. For example, most of the time, when the robot speaks to the human, it will expect them to look at it. A metric as the one described in [15], the "with-me-ness", would be suitable for the measurement of the human behavior tallying or not with the robot expectation. We plan to integrate it to our system in future work.

\section{B. Metrics related to effectiveness}

One can elaborate metrics to measure how well a task or an action is achieved. As discussed in [10], there are a variety of metrics that can be used.

As defined in [9] for navigation tasks, one can measure for different kinds of tasks, the ratio of successful ${ }^{1}$ executions to the total number of executions and the deviation from the initial plan (distance, cost, trajectory, etc).

We define here below three metrics allowing to measure the current (as opposed to overall) subtask/action effectiveness. Two of them are more suitable to geometric-based subtasks/actions being the distance to goal variation and the

\footnotetext{
${ }^{1}$ Obviously, the successfulness is context and task dependent and should be defined according to the needs
}

time to goal variation and, the other one is for non geometricbased subtasks/actions being the On-Time-Execution.

1) Variation of the distance to goal for geometricbased subtasks/actions: When one agent is performing a geometric-based subtask/action, observing if the agent is getting closer to the target position over time is a precious information giving a good hint about how well the subtask/action is going. Therefore, we introduce the distance to goal variation $\Delta D t G$ metric.

Let us assume that we can estimate any time $t$ the length of the shortest path to the goal path_length $(t)$. This can be given by a reactive motion planner [16]. If at time $t$ the agent is closer from its final position than at $t-1$, the variation of the distance to goal decreases or remains equal to 0 . Now, if the agent has not moved or is even further, the variation of the distance to goal increases. Then, we have defined the distance to goal variation as:

$$
\left\{\begin{array}{c}
\Delta D t G(t)=\max (0, \Delta D t G(t-1)-1) \\
\text { if path_length }(t)<\text { path_length }(t-1) \\
\Delta D t G(t)=\Delta D t G(t-1)+1, \text { otherwise }
\end{array}\right.
$$

2) Time to goal variation: This measure is intended to estimate the progress of a given task/action towards its goal in terms of the estimation of the time needed to reach it. It compares the current estimated time to goal with the initial estimated time to goal taking into account the current task duration. As so, it is possible to measure the variation compared to the initial plan. We define the time to goal variation $\Delta T t G$ as:

$$
\Delta T t G(t)=\max \left(0, e(t)+T t G(t)-T t G\left(T_{0}\right)\right)
$$

with $e(t)=t-T_{0}$ the task execution duration (time elapsed since the beginning of the task), $\operatorname{Tt} G(t)$ the current time to the goal, and $\operatorname{Tt} G\left(T_{0}\right)$ the initial planned time to goal.

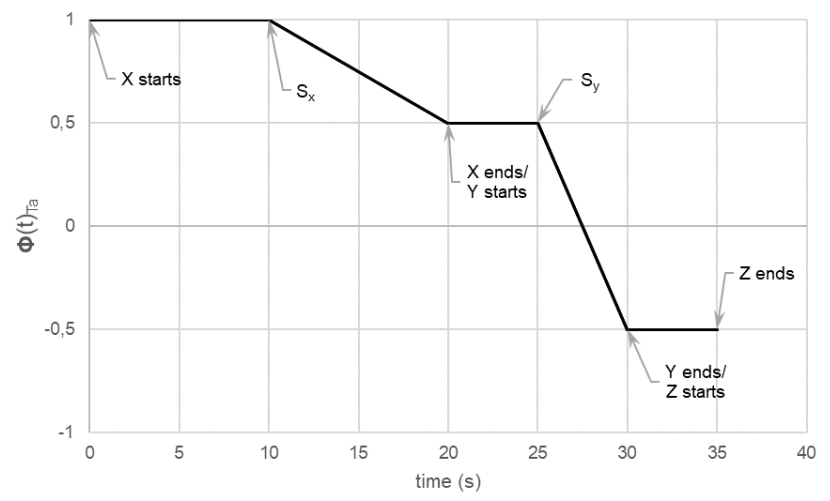

Fig. 1. Plot of $\Phi(t)_{T a}$ for a task composed of a sequence of three subtasks $X, Y, Z$ with $S D_{X}=10 s, V_{X}=0.5, S D_{Y}=5 s, V_{Y}=1, S D_{Z}=10 s$ and $V_{Z}=1$. The duration of $X$ exceeded $S D_{X}=10 \mathrm{~s}$ and reached $20 s$, the duration of $Y$ exceeded $S D_{Y}=5 s$ and reached $10 s$, finally the duration of $Z$ was less than $S D_{Z}=10 \mathrm{~s}$

3) On-Time-Execution: We introduce here the On-TimeExecution metric $\phi$ for subtasks/actions and the On-TimeExecution metric $\Phi$ for a whole task. This measure is 
intended to represent the degradation of the quality of execution of a HR task when its duration exceeds a certain time. To each subtask/action $a_{i}$, we associate two attributes whose values are defined by the designer: a soft deadline $S D_{i}$ and a decreasing quality speed $V_{i}$. If, at time $t$, the execution duration $e(t)=t-T_{0}$ of a substask or action $a_{i}$ which has started at $T_{0}$ exceeds $S D_{i}$, the quality will decrease over time at speed $V_{i}$ :

$$
\phi(t)_{i}=\max \left(V_{i} * \frac{-\max \left(e(t)-S D_{i}, 0\right)}{S D_{i}}+\alpha,-1\right)
$$

where $\alpha$ is the value initial value of $\phi_{i}$, when the subtask/action $a_{i}$ starts.

Then, we define a metric $\Phi$ for a task. It is an aggregation of the $\phi_{i}$ computed for each performed subtask/action $a_{i}$ of the task. At any moment, $\Phi$ can be seen as a memory of the previous steps, so the initial value $\alpha$ of $a_{i}$ is equal to the final value of $\phi_{i-1}$ of the previous subtask/action $a_{i-1}$, $\alpha=\phi\left(T_{\text {final }}\right)_{i-1}$. An example is given in Figure 1 .

We can notice that it is not possible for this metric to increase over time since it memorizes the values of the previous actions. However, the total computed QoI can get higher thanks to the other metrics. Moreover, $\phi$ can be used independently of $\Phi$. In such a case, the initial of value $\alpha$ of $\phi$ can be set to 1 .

\section{Metrics AgGREgATION PROCESS}

The metrics need to have their values normalized in order to be aggregated. We chose to have their output $\in\{-1,1\}$. A function is applied to a metric according to the need and depending on the context and the task. The choice should be based on:

- does the metric have a bounded value ?

- what value of the metric has a negative, neutral or positive impact?

\section{A. Normalization}

We defined a normalization function which can be used for values belonging to a bounded set, i.e., measures for which the minimum and maximum values are known. It is to apply in cases for which a measure approaching the boundary value $b_{1}$ has a negative impact on the quality evaluation whereas a measure approaching $b_{2}$ has a positive one. It allows to scale a measure $x$ between -1 and 1 :

$$
n(x)=2 * \frac{x-b_{1}}{b_{2}-b_{1}}-1
$$

Other normalization functions can be defined for measures having only a positive or a negative impact on the QoI.

\section{B. Logarithmic-based function}

We defined a parametric function fitting our needs. It allows to represent the evolution of a measure $x$, with $x \in\{0,+\infty\}$ ( $x$ being an amount for example). The function is defined as:

$$
s(x)=1-2 \exp \left(-\ln (2)\left(\frac{x}{t h}\right)^{k}\right), x>0
$$

with $s(x) \in\{-1,1\}$, th the parameter allowing to adjust the value for which the function returns 0 i.e., $s(t h)=0$ and, $k$ the parameter shaping the curve of the function. The variation is faster around th. The function $s(x)$ is illustrated in Figure 2 by one example.

Two similar functions can be used, $s_{p}$ for measures to be scaled between 0 and 1 with $s(t h)=0.5$ and $s_{n}$ for the ones between -1 and 0 with $s(t h)=-0.5$. They are defined as:

$$
\begin{aligned}
& s_{p}(x)=1-\exp \left(-\ln (2)\left(\frac{x}{t h}\right)^{k}\right), x>0 \\
& s_{n}(x)=-s_{p}(x)
\end{aligned}
$$
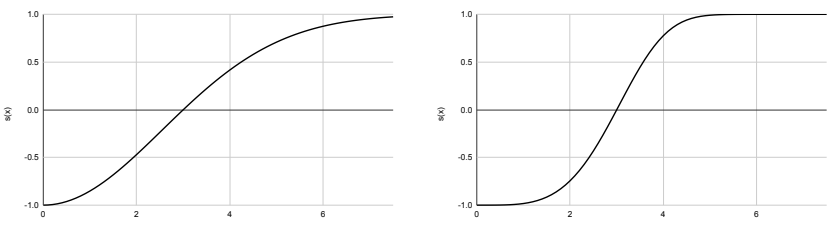

(a) Plot of $s(x)$ with $t h=3$ and (b) Plot of $s(x)$ with $t h=3$ and $k=2$ $k=4$

Fig. 2. Examples of $s(x)$ with two values of $k$

\section{IMPLEMENTATION OF THE QOI EVALUATOR FOR A GUIDING TASK}

As a proof-of-concept, we have implemented the Quality of Interaction Evaluator as part of a robotic system developed in the context of the MuMMER European project ${ }^{2}$. The project led to the deployment of a Pepper robot in a mall.

One of the core tasks for the robot consists in giving guidance to the customers to reach locations in the mall, by pointing at places and explaining the route to the wanted location. To do so, the robot is allowed to move in a limited area in order to place itself in a configuration where the landmarks it decides to indicate are visible to the human. It has been designed in accordance with human conventions based on a human-human guidance study conducted in the same environment [17].

In this first implementation of the Quality of Interaction Evaluator, we measured the interaction quality at the guiding task level and at the elementary action level. We provide and discuss in VII, a comparison of the QoI computed by the robot when it is dealing with an "ideal" human, a confused human and a not-compliant human. The experiment with these three different behaviors has been conducted in our lab.

\section{A. Guiding task description}

The guiding task is represented in Figure 3 as a hierarchical task network. The plan of the guiding task is a linear sequence of interactive subtasks to be executed one after the other:

\footnotetext{
${ }^{2} \mathrm{http}: / /$ mummer-project.eu/
} 


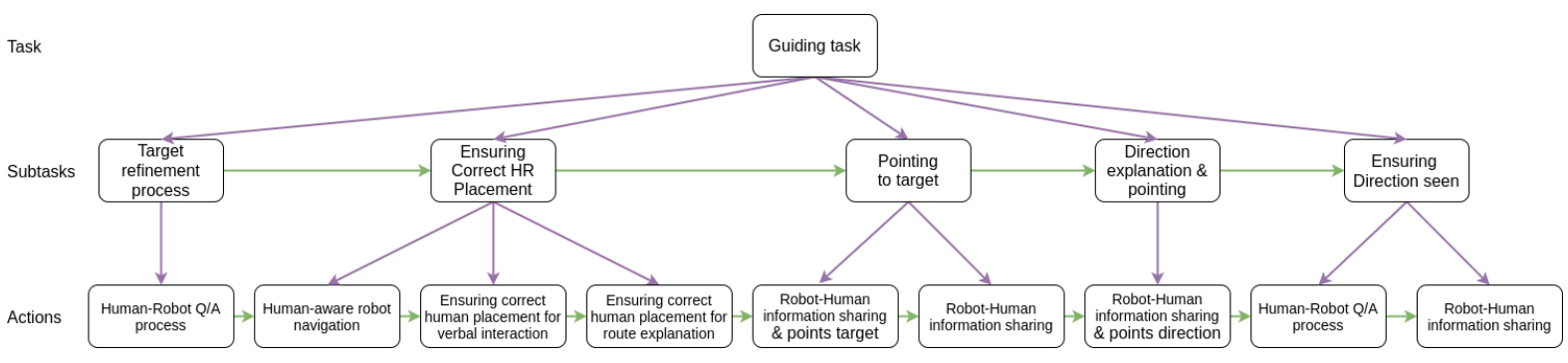

Fig. 3. The representation of the guiding task as a hierarchical task network with task, substasks and actions levels, exhibiting its incremental refinement into a sequence of HR interactive actions. Decomposition links are drawn in purple and the causal links are in green.

- Target refinement process: The robot tries, through dialog, to determine precisely for which place it has to give direction.

- Ensuring Correct HR Placement (optional): The robot and the human might move to better suited positions for pointing and seeing the target. This subtask is illustrated in Figure 4.

- Pointing to target: The robot points in the shop direction, along with a brief sentence.

- Direction explanation and pointing (optional): If there is one, the robot points at the first access point while verbalising the route explanation.

- Ensuring Direction seen or Route understood: The robot checks that the route direction has been understood by asking the person about it or if the first access point is visible, by asking if it has been seen.
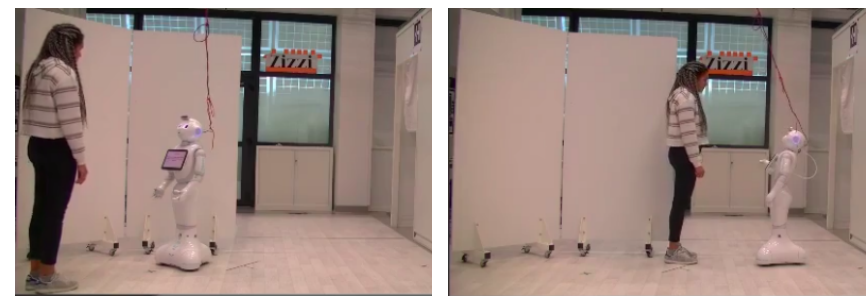

(a) Initial positions of the human and (b) The robot and the human are in the robot. The human asked the robot their final positions as planned by the where is the shop "Zizzi" (not visible robot. The robot is pointing to the to them because of the panels). shop direction which is now visible to them.

Fig. 4. The robot and the human might move from their initial positions to reach two which are better suited for pointing and seeing the target. So the robot navigates to its computed position, then waits and (if needed) asks the human to come to it. When the human is perceived, if the robot observes that they are not placed such as it can see the target, it will try to indicate to them verbally where to position themselves.

\section{B. Actions description}

The guiding task is performed as a sequence of HR interactive actions. It involves several turn-taking steps, the robot asking complementary information, informing the human or expecting an action or reaction from them. The different actions involved in the task are described below.

1) Robot-Human information sharing: The robot speaks to the human, shares information such as the route direction and announces the next step of the plan. It expects the human to be attentive, which means staying close enough and looking at the robot most of the time as a cue for listening.

2) Human-Robot Q/A process: As when sharing an information, the robot expects the human to be attentive. It also expects an answer which should be understandable and related to the question. If the given answer is out of topic or not understood by the robot, it will ask the human to repeat specifying that it has not understood.

3) Ensuring that Human moves aside: This action is used if, for pointing, the robot decides to place itself in a position which is very close to where the human is currently standing. In this case, the robot asks the human to step aside on the right or left, depending on the human's future position. If the human does not move or does not go far enough from the robot position, the robot repeats.

4) Human-aware robot navigation: The robot has to move from its initial position to its computed one. It navigates while respecting social constraints and its path may change as it adapts according to what the human is doing.

5) Ensuring correct human placement for verbal interaction: After it has moved, the robot asks the human to come in front of it. If the human is not perceived after a few seconds, the robot will ask again.

6) Ensuring correct human placement for route explanation: Once the human is in the robot field of view after the HR motion, they may not be at the right place to properly see what the robot has to point at. In this case, the robot will ask the human to move forward or backward if an object occludes the view for the human. This will stop if the robot computes that the position of the human allows them to see the target.

\section{Brief system description}

The overall architecture running on the robot is described in [18]. The Quality of Interaction Evaluator is part of the supervision system which is programmed in Jason [19], a BDI agent-oriented framework. The supervision system handles the HR collaborative task execution through Jason reactive plans. Throughout the task, the robot supervises the execution and adapts its responses to contingencies. The Quality of Interaction Evaluator is integrated into a Jason function (the reasoning cycle) which is invoked periodically. After multiple testings, we chose to have the Evaluator computing the QoI for both levels every second. Therefore, 
every second, the system computes the value of each metric and then outputs a value for $Q o I_{t a s k}$ and $Q o I_{a c t i o n}$.

\section{Selected metrics to evaluate the QoI at the task level}

The evaluation of the QoI has been integrated in the supervision system based on Jason. We selected the metrics for the guiding task among the ones defined in Section IV. The choice has been made according to the the context, the task and/or actions characteristics and the available data.

1) On-Time-Execution: The task is a sequence of subtasks with an associated soft deadline from which the QoI decreases, i.e. if the subtask lasts longer than expected. That is why we chose to select the On-Time-Execution metric as defined in the equation (4) as a metric for this task. For each subtask $i \in\{0,6\}$, we determined the values of the subtasks soft deadlines $S D_{i}$ using empirical data from numerous experiments with naive users as illustrated in Figure 5. We computed the average time execution of each subtask, after removing the cases for which the execution of the subtask was annotated as not smooth. Finally, we chose $V_{i}=0.5$ for all the subtasks.
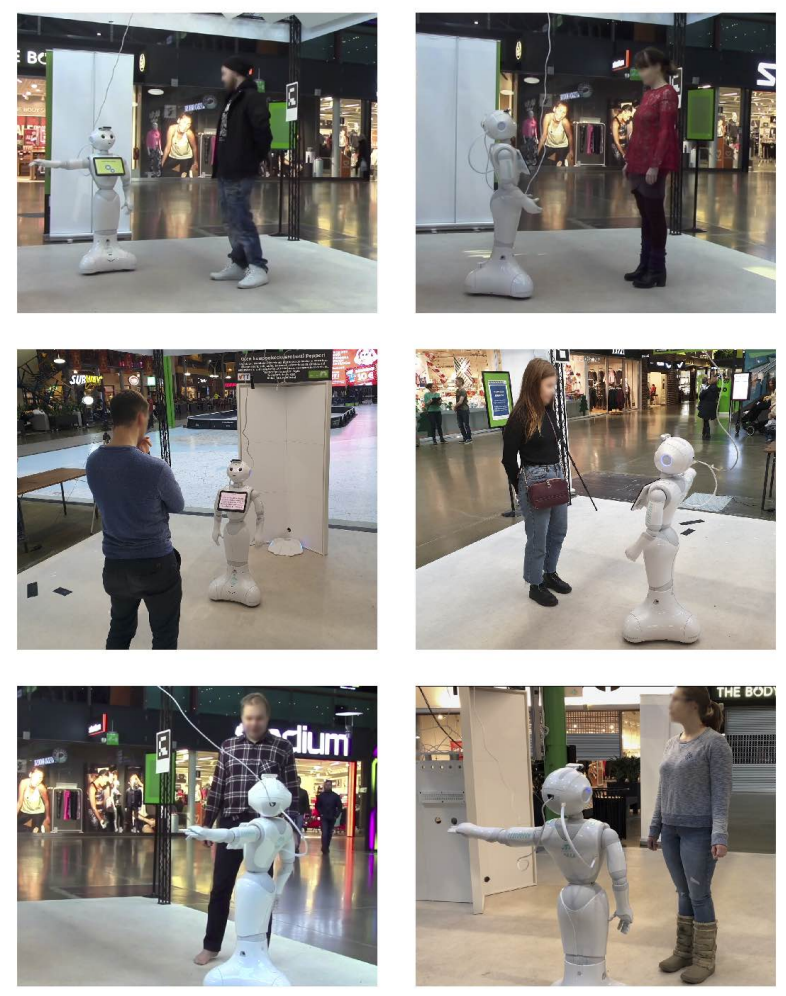

Fig. 5. The MuMMER robot in the Ideapark mall in Lempäälä, Finland. It is engaged in Route guiding task. Around 350 trials with dozens of customers in the mall allowed us to acquire experience, to draw lessons and to gather a set of empirical data in order to select the metrics and tune the measuring functions.

2) Actions QoI: The task QoI is also dependent of the actions QoI. That is why, besides the On-Time-Execution measures, we take into account the average QoI of the actions already executed or still running.

\section{E. Computation of the QoI at the task level}

The QoI for the task level is a combination of the OnTime-Execution metric and the actions QoI. After multiple trials with people, we have chosen different weights for those two elements, which gives:

$$
Q o I_{\text {guiding_task }}=\frac{\Phi_{\text {guiding_task }}+3 * \overline{Q o I}_{\text {actions }}}{4}
$$

\section{F. Selected metrics to evaluate the QoI at the action level}

1) Human attention monitoring (Fulfilling robot expectations): When speaking to a human, the robot expects that they are paying attention to it. Therefore, we measure a kind of attention ratio i.e., the time during which the human is attentive to the robot when it speaks compared to the total time of the speech. The ratio is scaled with the formula (5) to fit between -1 and 1 . As the minimum value for this ratio is 0 and the maximum value is 1 , then $b_{1}=0$ and $b_{2}=1$. Therefore, if $A R=0$ or $A R=1$, we will respectively have $Q o I_{H A}=-1$ or $Q o I_{H A}=1$.

$$
\left\{\begin{array}{l}
Q o I_{H A}=n(A R), \text { with } b_{1}=0 \text { and } b_{2}=1 \\
A R=\frac{\text { duration } \text { isAttentiveTo(robot })=\text { true }}{\text { duration }_{\text {robot_speaks }}}
\end{array}\right.
$$

2) Action efficiency (Human contribution to the goal): When expecting an action from the human, the robot verbalizes its needs. If the action is not executed or not correctly performed, the robot will ask again, up to 3 times, that is why we set $b_{1}=3$ and $b_{2}=0$. It means that the action efficiency decreases based on the number of trials. Therefore we choose the equation (5) to represent this behavior in order to have $Q O I_{A E}=1$ for $n b_{-}$trials $=0$ and $Q O I_{A E}=0$ for nb_trials $=3$.

$$
Q o I_{A E}=n\left(n b_{-} \text {trials }\right), \text { with } b_{1}=3 \text { and } b_{2}=0
$$

3) Time to goal variation: When the robot navigates, its path can change according to human motions close to it. To estimate if things are going well, we measure the time to goal variation. If the effective execution duration is equal to what was planned (or even less), $\Delta T t G$ remains to 0 and $Q o I_{\Delta T t G}$ should be 1 . Now, if $\Delta T t G$ increases because of non-anticipated contingencies, then $Q_{o} I_{\Delta T t G}$ should decrease. However, we do not know the maximum value that $\Delta T t G$ can have. Therefore, based on these characteristics, we give the time to goal variation of the equation (3) as input of the opposite of the equation (6).

$$
Q o I_{\Delta T t G}=-s(\Delta T t G) \text {, with } t h=5 \text { and } k=1.5
$$

4) Distance to goal variation: When the robot asks the human to come closer or make a step aside, we measure how the human moves forward or moves away from the future robot position. In the first case, the human has to come closer from a computed position. Therefore, we give the distance to goal variation defined in the equation (2) as input of the opposite of the equation (6). Indeed, similarly to the time to goal variation, the closest to 0 the distance to goal variation is, the better.

$$
Q o I_{\Delta D t G}=-s(\Delta D t G), \text { with with } t h=5 \text { and } k=1.5
$$


In the second case, if at time $t$, the human is further from the planned robot position than at $t-1$, the distance to goal variation decreases or stays equal to 0 . However, if the human has not moved or is closer, the distance to goal variation increases. Then, the distance to goal variation defined in equation (2) (but with adapted conditions for the geometric distance as the human needs to get further from a position and not closer) is given as input of the opposite of the equation (6), as the closest to 0 the distance to goal variation is, the better. So in (2), the condition is the inverse of the one in the original equation:

$$
\left\{\begin{array}{c}
\Delta D t G(t)=\max (0, \Delta D t G(t-1)-1) \\
\text { if } d(p c(t), p g(t))>d(p c(t-1), p g(t-1)) \\
\Delta D t G(t)=\Delta D t G(t-1)+1, \text { otherwise }
\end{array}\right.
$$

\section{G. Computation of the QoI for each actions}

For each action, a QoI is computed from the metrics described in VI-F. A summary is given in table I.

\section{RESUlTS}

As a first step of validation for our approach, we measured the QoI of the guiding task with three different human behaviors:

\begin{tabular}{|c|c|}
\hline Action & Action QoI \\
\hline Robot-Human information sharing & $Q o I_{H A}$ \\
\hline Human-Robot Q/A process & $\left(Q o I_{H A}+Q o I_{A E}\right) / 2$ \\
\hline Ensuring that Human moves aside & $\left(Q o I_{\Delta D t G}+Q o I_{A E}\right) / 2$ \\
\hline Human-aware robot navigation & $Q o I_{\Delta T t G}$ \\
\hline $\begin{array}{l}\text { Ensuring correct } \mathrm{H} \text { placement for verbal } \\
\text { interaction }\end{array}$ & $Q o I_{A E}$ \\
\hline $\begin{array}{c}\text { Ensuring correct } \mathrm{H} \text { placement for route } \\
\text { explanation }\end{array}$ & $\left(Q o I_{\Delta D t G}+Q o I_{A E}\right) / 2$ \\
\hline
\end{tabular}

TABLE I

QOI COMPUTATION FOR THE ACTIONS OF THE GUIDING TASK

1) a human executing perfectly the expected actions and not disturbing the robot when it navigates (i.e. the 'ideal' human from the robot point of view)

2) a human a bit confused, trying to contribute to the task success but not doing everything well

3) a human willing to disturb the robot during the task

In the three cases, the task was conducted until its end. The measured QoI for behaviors 2 and 3 is presented in Figure 6. One may note that the QoI measured over time effectively degrades when the human does not contribute or even voluntarily disturbs the task execution.

We do not illustrate the case 1 as the interaction is very

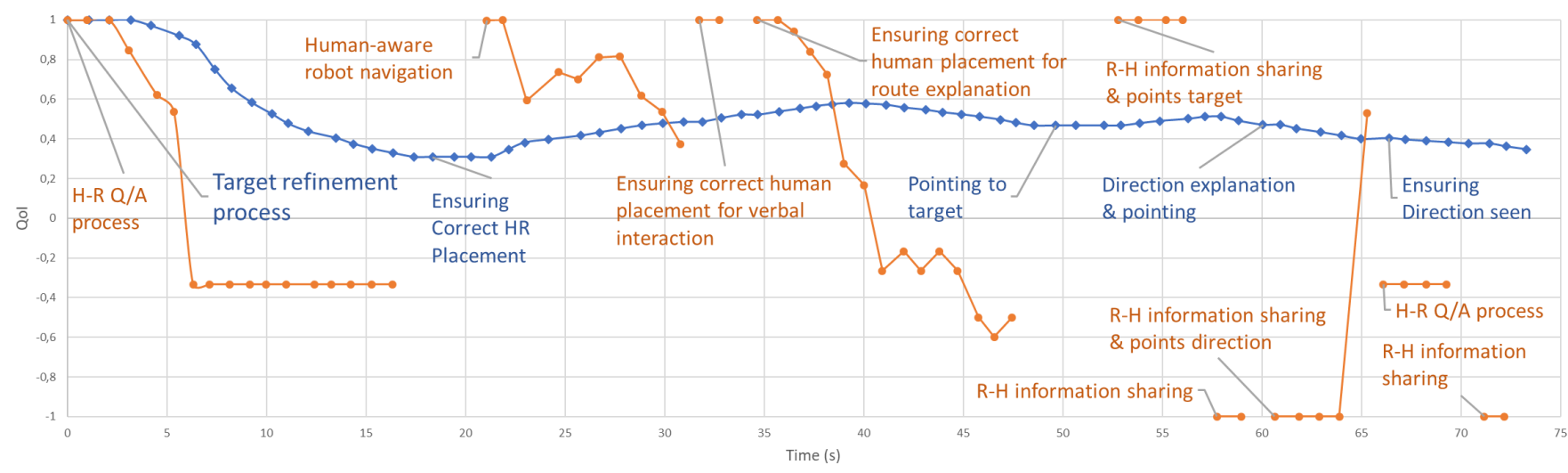

(a) Evolution over time of the measured QoI for the confused human (behavior 2).

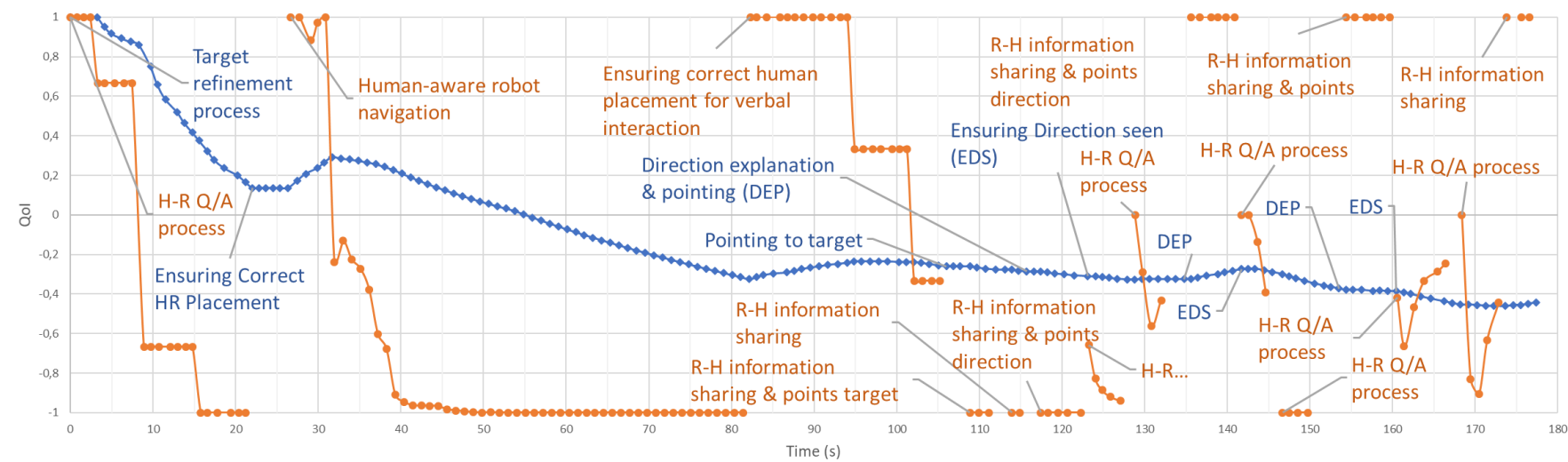

(b) Evolution over time of the measured QoI for the annoying human (behavior 3).

Fig. 6. Evolution over time of the measured QoI for the route guidance task with the human behaviors 2 and 3. The QoI for the task is drawn in blue, and the QoI for the actions is drawn in orange. 
smooth since the human behaves as the robot expects during the whole task. Therefore, the task and action QoIs remain with the highest value, 1, all along. In Figure 6a, the human is, from time to time, not very attentive. Also, they gave a first answer not understood by the robot at the first question and then took a small time before answering. Then, they prevented the robot from moving as it had planned and once the robot reached its position, they took time to come as close as the robot wanted. Finally, in Figure $6 b$, the human gave three incomprehensible answers at the first question, blocked multiple times the robot in its movement, waited for the robot to ask twice to come in front of it and finally asked the robot to point and explain the route three times. In this last example, the QoI decreases all along the interaction as nothing is going very well, unlike the second example for which the QoI does not drop too much even if the robot has experienced some unexpected events and human reactions.

\section{CONCLUSION AND FUTURE WORK}

We introduced a novel way to endow the robot with the ability to evaluate its human-robot interaction experience. We proposed metrics to be used by a measurement process integrated in a cognitive and collaborative robot. Our system computes and estimates in real-time the Quality of Interaction while performing collaborative tasks with humans. This evaluation is based on multiple metrics chosen and combined according to the needs of the application. The set of metrics proposed can be and will be incrementally refined.

As a proof-of-concept, we implemented a first version of such system and tested it on an interactive robot dedicated to provide route guidance to customers in a large mall. The approach gave satisfactory results. It showed the potential ability of the robot to detect momentary decreases of the Quality of Interaction and also more serious degradation of it which may need drastic changes of behavior for the robot.

In our agenda, we plan to conduct user studies where we will ask humans to evaluate the quality of their interaction with the robot in a similar manner. The goal will be to analyse and compare this to the evaluation of the interaction quality estimated by our robot.

Finally, we intend to exploit this QoI evaluation process in order to allow the robot to "close the loop" and smoothly adapt it decisions and execution modalities and also to detect if the human is trying to pull the robot strings. In such a situation, the robot can take the decision to disengage or even recall to the human their duties as a partner.

\section{ACKNOWLEDGMENTS}

Many thanks to Michaël Mayer for his technical expertise and his help on the mathematical formalization.

This work has been funded by the French Agence Nationale de la Recherche JointAction4HRI project ANR16-CE33-0017 and the Artificial and Natural Intelligence Toulouse Institute (ANITI).

\section{REFERENCES}

[1] S. Devin and R. Alami, "An implemented theory of mind to improve human-robot shared plans execution," in The Eleventh ACM/IEEE International Conference on Human Robot Interaction, ser. HRI '16. IEEE Press, 2016, p. 319-326.

[2] S. Lemaignan, M. Warnier, E. A. Sisbot, A. Clodic, and R. Alami, "Artificial Cognition for Social Human-Robot Interaction: An Implementation," Artificial Intelligence, vol. 247, pp. 45-69, June 2017.

[3] J. R. Movellan, F. Tanaka, I. R. Fasel, C. Taylor, P. Ruvolo, and M. Eckhardt, "The rubi project: A progress report," in 2007 2nd ACM/IEEE International Conference on Human-Robot Interaction (HRI), 2007, pp. 333-339.

[4] G. Hoffman, "Evaluating fluency in human-robot collaboration," IEEE Transactions on Human-Machine Systems, vol. 49, no. 3, pp. 209-218, June 2019.

[5] Shuyin Li, M. Kleinehagenbrock, J. Fritsch, B. Wrede, and G. Sagerer, "'biron, let me show you something": evaluating the interaction with a robot companion," in 2004 IEEE International Conference on Systems, Man and Cybernetics (IEEE Cat. No.04CH37583), vol. 3, 2004, pp. 2827-2834 vol.3.

[6] K. Dautenhahn and I. Werry, "A quantitative technique for analysing robot-human interactions," in IEEE/RSJ International Conference on Intelligent Robots and Systems, vol. 2, Sep. 2002, pp. 1132-1138 vol.2.

[7] A. Weiss, R. Bernhaupt, M. Lankes, and M. Tscheligi, "The usus evaluation framework for human-robot interaction," in Adaptive and Emergent Behaviour and Complex Systems - Proceedings of the 23rd Convention of the Society for the Study of Artificial Intelligence and Simulation of Behaviour, AISB 2009, 12 2009, pp. 158-165.

[8] D. Feil-Seifer, K. Skinner, and M. Matarić, "Benchmarks for evaluating socially assistive robotics," Interaction Studies, vol. 8, pp. 423439, 102007.

[9] A. Steinfeld, T. Fong, D. Kaber, M. Lewis, J. Scholtz, A. Schultz, and M. Goodrich, "Common metrics for human-robot interaction," in Proceedings of the 1st ACM SIGCHI/SIGART Conference on Humanrobot Interaction, ser. HRI '06. New York, NY, USA: ACM, 2006, pp. $33-40$.

[10] D. R. Olsen and M. A. Goodrich, "Metrics for evaluating human-robot interaction," in PERMIS, Gaithersburg, MD, USA, 2003.

[11] A. Tanevska, G. Rea, F.and Sandini, and A. Sciutti, "Towards an Affective Cognitive Architecture for Human-Robot Interaction for the iCub Robot," in 1st Workshop on "Behavior, Emotion and Representation: Building Blocks of Interaction", Bielefeld, Germany, Oct. 2017.

[12] K. Itoh, H. Miwa, Y. Nukariya, M. Zecca, H. Takanobu, S. Roccella, M. C. Carrozza, P. Dario, and A. Takanishi, "Development of a bioinstrumentation system in the interaction between a human and a robot," in 2006 IEEE/RSJ IROS, Oct 2006, pp. 2620-2625.

[13] F. Dehais, E. A. Sisbot, R. Alami, and M. Causse, "Physiological and subjective evaluation of a human-robot object hand-over task," Applied Ergonomics, vol. 42, no. 6, pp. 785-791, Nov. 2011.

[14] S. Bensch., A. Jevtić., and T. Hellström., "On interaction quality in human-robot interaction," in Proceedings of the 9th International Conference on Agents and Artificial Intelligence - Volume 1: ICAART,, INSTICC. SciTePress, 2017, pp. 182-189.

[15] S. Lemaignan, F. Garcia, A. Jacq, and P. Dillenbourg, "From real-time attention assessment to "with-me-ness" in human-robot interaction," in 2016 11th ACM/IEEE International Conference on Human-Robot Interaction (HRI), 2016, pp. 157-164.

[16] H. Khambhaita and R. Alami, "Viewing robot navigation in human environment as a cooperative activity," in Robotics Research, N. M. Amato, G. Hager, S. Thomas, and M. Torres-Torriti, Eds. Cham: Springer International Publishing, 2020, pp. 285-300.

[17] K. Belhassein, A. Clodic, H. Cochet, M. Niemelä, P. Heikkilä, H. Lammi, and A. Tammela, "Human-Human Guidance Study," Dec. 2017.

[18] M. E. Foster, B. Craenen, A. Deshmukh, O. Lemon, E. Bastianelli, C. Dondrup, I. Papaioannou, A. Vanzo, J.-M. Odobez, O. Canévet, Y. Cao, W. He, A. Martínez-González, P. Motlicek, R. Siegfried, R. Alami, K. Belhassein, G. Buisan, A. Clodic, A. Mayima, Y. Sallami, G. Sarthou, P.-T. Singamaneni, J. Waldhart, A. Mazel, M. Caniot, M. Niemelä, P. Heikkilä, H. Lammi, and A. Tammela, "Mummer: Socially intelligent human-robot interaction in public spaces," in AAAI Fall Symposium Series 2019, November 2019.

[19] R. H. Bordini, J. F. Hübner, and M. Wooldridge, Programming MultiAgent Systems in AgentSpeak Using Jason (Wiley Series in Agent Technology). USA: John Wiley \& Sons, Inc., 2007. 